\title{
A Glutamate-Substituted Mutant Mimics the Phosphorylated and Active Form of Guanylyl Cyclase-A
}

\author{
Neil M. Otto, William G. McDowell, Deborah M. Dickey, and Lincoln R. Potter \\ Department of Biochemistry, Molecular Biology, and Biophysics, University of Minnesota Medical School, \\ Minneapolis, Minnesota
}

Received December 20, 2016; accepted April 4, 2017

\section{ABSTRACT}

Multisite phosphorylation is required for activation of guanylyl cyclase (GC)-A, also known as NPR-A or NPR1, by cardiac natriuretic peptides (NPs). Seven chemically identified sites (Ser487, Ser-497, Thr-500, Ser-502, Ser-506, Ser-510, and Thr-513) and one functionally identified putative site (Ser-473) were reported. Single alanine substitutions for Ser-497, Thr-500, Ser-502, Ser-506, and Ser-510 reduced maximal velocity $\left(V_{\max }\right)$, whereas glutamate substitutions had no effect or increased $V_{\max }$. Ala but not Glu substitution for Ser-497 increased the Michaelis constant $\left(K_{\mathrm{m}}\right)$ approximately $400 \%$. A GC-A mutant containing Glu substitutions for all seven chemically identified sites (GC-A-7E) had a $K_{\mathrm{m}}$ approximately 10-fold higher than phosphorylated wild-type (WT) GC-A, but one additional substitution for Ser-473 to make GC-A-8E resulted in the same $V_{\max }$,
$K_{\mathrm{m}}$, and $\mathrm{EC}_{50}$ as the phosphorylated WT enzyme. Adding more glutamates to make GC-A-9E or GC-A-10E had little effect on activity, and sequential deletion of individual glutamates in GC-A$8 \mathrm{E}$ progressively increased the $K_{\mathrm{m}}$. Double Ala substitutions for Ser-497 and either Thr-500, Ser-510 or Thr-513 in WT-GC-A increased the $K_{\mathrm{m}} 23$ - to 70 -fold but the same mutations in GC-A$8 \mathrm{E}$ only increased the $K_{\mathrm{m}}$ 8-fold, consistent with one site affecting the phosphorylation of other sites. Phosphate measurements confirmed that single-site Ala substitutions reduced receptor phosphate levels more than expected for the loss of a single site. We conclude that a concentrated region of negative charge, not steric properties, resulting from multiple interdependent phosphorylation sites is required for a GC-A conformation capable of transmitting the hormone binding signal to the catalytic domain.

\section{Introduction}

Atrial natriuretic peptide (ANPs) and B-type natriuretic peptides (NPs) are cardiac hormones that reduce blood pressure and volume by activating guanylyl cyclase (GC)-A, the target of two drugs approved by the U.S. Food and Drug Administration (Hubers and Brown, 2016). GC-A is a homomultimeric, single membrane-spanning enzyme that catalyzes the conversion of GTP to the second messenger, cGMP and pyrophosphate. GC-A contains a glycosylated extracellular ligand binding domain, a single membrane-spanning region, and a multidomain intracellular region consisting of a kinase homology domain (KHD), a dimerization domain, and a C-terminal GC catalytic domain (Potter and Hunter, 2001). The homologous enzyme called GC-B is 78\% identical to GC-A at the intracellular amino acid level but is activated by C-type natriuretic peptide (CNP), which regulates long bone growth, axonal bifurcation, and resumption of meiosis in the oocyte (Potter, 2011; Kuhn, 2016).

GC-A is maximally phosphorylated in serum-starved 293 cells (Potter and Garbers, 1992; Koller et al., 1993; Joubert et al., 2001). Prolonged ANP exposure or brief exposure to chemical activators of protein kinase $\mathrm{C}$ such as lysophosphatidic acid cause GC-A dephosphorylation, which reduces ANP-dependent

This research was supported in part by the National Institutes of Health National Institute of General Medical Sciences [Grant R01GM098309] and by a grant from the Fund for Science.

https://doi.org/10.1124/mol.116.107995.
GC activity (Potter and Garbers, 1992, 1994; Koller et al., 1993; Joubert et al., 2001; Müller et al., 2006). Prolonged CNP exposure, or brief exposure to protein kinase $\mathrm{C}$ activators or intracellular calcium-elevating agents, results in GC-B dephosphorylation and inactivation as well (Potter, 1998; Potter and Hunter, 2000; Abbey and Potter, 2002, 2003; Abbey-Hosch et al., 2004; Egbert et al., 2014). Endogenous GC-A and GC-B isolated from normal mouse and rat tissues are also phosphorylated (Bryan et al., 2006). Congestive heart failure is associated with increased GC-B and reduced GC-A activity, but the role of dephosphorylation in the latter process is unclear (Bryan et al., 2007; Dickey et al., 2007, 2012). In contrast, luteinizing hormone-dependent inactivation of GC-B was correlated with dephosphorylation of GC-B in rat ovarian follicles (Egbert et al., 2014), and CNP-dependent GC activity in ovarian follicle membranes from a "knock-in" mouse expressing a mutant form of GC-B containing glutamate substitutions for all known phosphorylation sites was resistant to luteinizing hormone-dependent inactivation (Shuhaibar et al., 2016).

Seven GC-A phosphorylation sites and six homologous phosphorylation sites in GC-B were chemically identified in the N terminus of the KHD (Fig. 1) (Potter and Hunter, 1998a; Schröter et al., 2010; Yoder et al., 2010, 2012). Alanine substitutions for all individual sites but Ser-487 in GC-A decreased ANP-dependent activity in GC assays using 1-mM GTP substrate concentrations. Strikingly, the mutation of four 
or more phosphorylation sites to alanine yielded enzymes that were completely unresponsive to ANP (Potter and Hunter, 1998b). Conversely, glutamate substitution mimicked the functional effect of a single phosphorylation site (Potter and Hunter, 1998b), but mutating all six sites to glutamate yielded an enzyme with only about $20 \%$ of the activity of the phosphorylated wild-type (WT) enzyme (Potter and Hunter, 1999; Yoder et al., 2012). In subsequent studies, the substitution of Glu but not Ala for Ser-473 in GC-A or Ser-489 in GC-B produced near WT-like enzymatic activity in single substrate assays but no peptides containing these juxtamembrane sites were identified from GC-A or GC-B by mass spectrometry or tryptic phosphopeptide labeling (Yoder et al., 2012).

Surprisingly, the effect of each individual phosphorylation site on the $V_{\max }$ and $K_{\mathrm{m}}$ of GC-A has not been examined. Here, we performed substrate-velocity assays on membranes prepared from 293 cells expressing Ala or Glu substitutions for individual or multiple phosphorylation sites to determine how each site (or multiple sites) affects enzymatic activity. As a result, we created a phosphomimetic mutant of GC-A that has the same enzymatic characteristics as the phosphorylated WT enzyme.

\section{Materials and Methods}

Reagents. ${ }^{125} \mathrm{I}$-cGMP radioimmunoassay kits were from PerkinElmer (Waltham, MA) and the NPs were from Sigma (St. Louis, MO).

Mutagenesis. The Ala or Glu substitutions for each phosphorylation site or multiple phosphorylation sites were created by the QuikChange II system (Stratagene, Cedar Creek, TX) on the CMV3GC-A plasmid as previously described (Potter and Hunter, 1998b).

Transient Transfection. Human embryonic kidney 293T cells were transiently transfected with $5 \mu \mathrm{g}$ pCMV3-GC-A plasmids containing single or multiple phosphorylation site mutations by the HEPES-calcium-phosphate precipitation method as previously described (Yoder et al., 2012).

Membrane Preparation. Cells cultured on $10-\mathrm{cm}$ plates were placed in serum-free media for 4 hours before membranes were prepared. Membranes were harvested at $4^{\circ} \mathrm{C}$ by washing the plates twice with phosphate-buffered saline, scraping the cells off the plates in $0.6 \mathrm{ml}$ phosphatase inhibitor buffer (PIB) (Antos et al., 2005). Cells were lysed by sonication and the lysates were centrifuged at $20,000 \times g$ for 15 minutes at $4^{\circ} \mathrm{C}$. The supernatant was aspirated and the membrane pellet was resuspended with $0.5 \mathrm{ml} \mathrm{PIB}$ and centrifuged again at $20,000 \times g$ for 15 minutes at $4^{\circ} \mathrm{C}$. The supernatant was aspirated, and the pellet was resuspended in PIB to yield a protein concentration between 1 and $3 \mathrm{mg} / \mathrm{ml}$. Crude membranes were assayed for GC activity without freezing.

GC Assays. Crude membranes were assayed for GC activity at $37^{\circ} \mathrm{C}$ in a buffer containing $0.5 \mathrm{mM}$ isomethylxanthine to inhibit

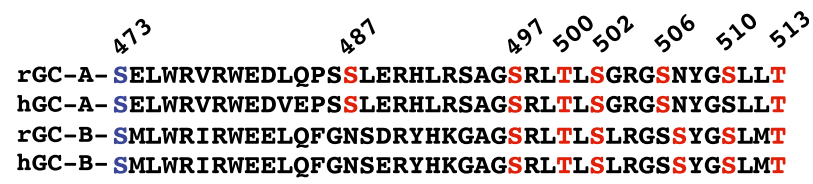

Fig. 1. Comparison of the primary amino acid sequence of the intracellular juxtamembrane region of rat and human GC-A and GC-B. Red residues are phosphorylation sites that have been identified by mass spectrometry or tryptic phosphopeptide mapping of ${ }^{32} \mathrm{PO}_{4}$ label enzymes isolated from living cells. The blue residue is a conserved serine and putative phosphorylation site, which was identified based on enzymatic changes associated with phosphomimetic substitutions. Sequences were aligned with Clustal software (version 2.0.1). Numbers correspond to residues from rat GC-A. hGC, human GC; rGC, rat GC. phosphodiesterases, $5 \mathrm{mM} \mathrm{MgCl}_{2}$, and a nucleotide regenerating system as reported (Robinson and Potter, 2012); 0.02 ml crude membranes were added per assay. A solution of $100 \mathrm{mM}$ GTP and $100 \mathrm{mM} \mathrm{MgCl} 2$ was diluted to the appropriate substrate concentrations used for each assay. Because enzymatic activity was not completely linear with time, the kinetic parameters are considered "apparent."

Immunoblot Analysis. Ten micrograms of membrane protein prepared in PIB from transiently transfected 293 cells was fractionated on an $8 \%$ gel by SDS-PAGE and blotted to a polyvinylidene fluoride membrane for 2 hours at $4^{\circ} \mathrm{C}$. The amount of GC-A was detected with rabbit polyclonal antiserum 6325 at a dilution of $1 / 10,000$ using a LI-COR detection system as described (LI-COR, Lincoln, NE).

Experimental Protocol. Plasmids encoding WT GC-A and corresponding Ala and Glu mutants were transiently transfected into 293 cells (Yoder et al., 2010). Two days later, crude membranes were prepared from sonicated cells in a buffer designed to maximally inhibit GC-A dephosphorylation (Antos et al., 2005) and were assayed for cGMP production for 5 minutes in the presence of $1 \mu \mathrm{M}$ ANP, $1 \mathrm{mM}$ ATP, and increasing concentrations of $\mathrm{Mg}^{2+}$ GTP to determine the $V_{\max }$ and $K_{\mathrm{m}}$ of the enzymes. The data from each set of WT, Ala mutant, and Glu single-mutant enzymes are presented in Table 1, which represents three separate experiments in which each substrate concentration was measured in duplicate $(n=3)$. The three individual membrane preparations were also Western blotted and the intensities for all enzymes were compared by a two-tailed $t$ test. None of the mutants were expressed at higher or lower levels than the other mutant or WT GC-A (data not shown). Since membranes from 293 cells transfected with a plasmid encoding GFP or empty vector contain less than $2 \%$ of the activity detected in membranes from cells transfected with WT-GC-A, the activities and protein levels detected were attributed to the transfected plasmids for each experiment (Yoder et al., 2010). Because the results for each phosphorylation site were generated from a separate transfection with differing transfection efficiencies, the data are presented as the percentage of WT activity for each experiment.

Whole-Cell cGMP Elevation Assays. Transiently transfected 293 cells grown in 48 -well plates to $90 \%$ confluence were pretreated for 10 minutes at $37^{\circ} \mathrm{C}$ in Dulbecco's modified Eagle's medium containing $25 \mathrm{mM}$ HEPES, $\mathrm{pH} 7.4$, and $0.5 \mathrm{mM}$ 1-methyl-3-isobutylxanthine to inhibit phosphodiesterases (Dickey et al., 2008). This medium was

\section{TABLE 1}

Effect of individual alanine or glutamate substitutions at GC-A phosphorylation sites on maximal velocity and the Michaelis constant of the enzyme

Values are presented as the mean \pm S.E.M. percentage of WT $(n=3)$.

\begin{tabular}{lcc}
\hline Mutation & $V_{\max }$ & $K_{\mathrm{m}}$ \\
\hline S497A & $59 \pm 8^{*}$ & $389 \pm 174^{*}$ \\
S497E & $119 \pm 3$ & $184 \pm 79$ \\
T500A & $45 \pm 4^{*}$ & $150 \pm 56$ \\
T500E & $93 \pm 7$ & $107 \pm 38$ \\
S502A & $70 \pm 9^{*}$ & $70 \pm 41$ \\
S502E & $107 \pm 12$ & $113 \pm 54$ \\
S506A & $87 \pm 4^{*}$ & $221 \pm 44^{*}$ \\
S506E & $119 \pm 4^{*}$ & $196 \pm 28^{*}$ \\
S510A & $58 \pm 11^{*}$ & $195 \pm 151$ \\
S510E & $113 \pm 14$ & $162 \pm 82$ \\
T513A & $80 \pm 8$ & $165 \pm 70$ \\
T513E & $152 \pm 10^{*}$ & $111 \pm 33$ \\
S487A & $107 \pm 14$ & $99 \pm 58$ \\
S487E & $130 \pm 16$ & $144 \pm 77$ \\
S473A & $91 \pm 3$ & $88 \pm 16$ \\
S473E & $83 \pm 8$ & $116 \pm 58$ \\
\hline
\end{tabular}

293 cells were transiently transfected with plasmids expressing the indicated mutant and assayed for GC activity in the presence $1 \mu \mathrm{M}$ ANP, $1 \mathrm{mM}$ ATP, and increasing concentrations of $\mathrm{Mg}^{2+}$ GTP. Average $V_{\max }$ and $K_{\mathrm{m}}$ values for WT GC-A were $16.5 \pm 3.9 \mathrm{nmol} \mathrm{cGMP} / \mathrm{mg}$ protein per 5 minutes and $54.8 \pm 1.4 \mu \mathrm{M} \mathrm{GTP}$, respectively.

$* P<0.0125$ (significantly different from WT-GC-A) 
aspirated and replaced with the same medium containing increasing concentrations of rat ANP for 1 minute. The reaction was stopped by aspiration of the medium and addition of $0.2 \mathrm{ml}$ ice-cold $80 \%$ ethanol. cGMP concentrations in the extract were determined by cGMP radioimmunoassay (Abbey and Potter, 2002).

Gel Staining. Eight-percent resolving gels were fixed in a $30-\mathrm{ml}$ solution of $50 \%$ methanol and $10 \%$ acetic acid for 30 minutes with gentle rocking. The solution was changed two times for a total of three washes in the fixing solution. The gels were then washed twice in $100 \mathrm{ml}$ water for 10 minutes. Ten milliliters of Pro-Q Diamond phosphoprotein gel stain was added, and the gels were incubated with gentle rocking for 1.5 hours in the dark. The gels were then destained with $80 \mathrm{ml}$ of a solution of $20 \%$ acetonitrile and $50 \mathrm{mM}$ sodium acetate ( $\mathrm{pH} 4.0)$ for 15 minutes. This wash was repeated two times for a total of three washes. The gels were then rinsed in water, scanned with a 532-nm laser, and imaged with Fuji FLA 5000 software (Fujifilm, Tokyo, Japan). After imaging, the same gels were stained with 50\% methanol, $7 \%$ acetic acid, and $0.1 \%$ Coomassie Brilliant Blue for 10 minutes and destained in a solution of 50\% methanol and $7 \%$ acetic acid.

Statistical Analysis. Statistical analyses were performed with GraphPad Prism 7 software (GraphPad Inc., La Jolla, CA). Substratevelocity curves were analyzed by nonlinear regression using a Michaelis-Menten model to determine the maximal velocity $\left(V_{\max }\right)$ and the Michaelis constant $\left(K_{\mathrm{m}}\right)$. For each pair of enzymes and each model parameter, we are comparing the full model fit against a null model in which that parameter set to be equal. Because of this, we used the extra sum of squares $F$ test to generate $P$ values. $P$ values were then adjusted using the Bonferroni correction for each set of four comparisons. Dose-response curves were analyzed by nonlinear regression using a sigmoidal dose-response curve to determine the $\mathrm{EC}_{50}$.

\section{Results}

Effect of Single Phosphorylation Site Substitutions. Ala mutants of the first six reported GC-A phosphorylation sites (Ser-497, Thr-500, Ser-502, Ser-506, Ser-510, and Thr513) have reduced ANP-dependent GC activity using single substrate concentration assays (Potter and Hunter, 1998b), but how the mutations affect the activity of the enzyme is not known. Here, we measured the effects of single Ala and Glu mutants with increasing substrate concentrations to characterize the effects on maximal velocity and the Michaelis constant as well as to determine the ability of the corresponding Glu mutation to mimic the functional effects of each individual phosphorylation site (Table 1). Every Ala substitution for the first six discovered sites reduced the $V_{\max }$ of GC-A, although the substitution at Thr-513 was not statistically significant. The reduction in $V_{\max }$ ranged from $13 \%$ to $55 \%$. In contrast, $V_{\max }$ values for the Glu substitutions for the corresponding residues were either not lower or higher than the $V_{\max }$ values obtained for WT-GC-A. Five of the six Ala substitutions increased the $K_{\mathrm{m}}$, but only the Ala mutations at Ser- 497 and Ser506 reached statistical significance. However, since both the Ala and Glu substitutions for Ser-506 increased the $K_{\mathrm{m}}$, the change in activity cannot be due to a charge difference.

The seventh GC-A phosphorylation site identified by mass spectrometry was Ser-487 (Schröter et al., 2010; Yoder et al., 2010). Initial single substrate concentration assays on the Ser-487-Glu mutant indicated reduced ANPdependent activity (Schröter et al., 2010). Subsequent assays confirmed the reduced activity for Glu- 487 but found no difference in activity between the WT enzyme and the Ala-487 mutant (Yoder et al., 2010). Here, substrate-velocity assays on mutants with single Ala or Glu substitutions for Ser-487 revealed no significant differences in the $K_{\mathrm{m}}$ or $V_{\max }$ between either mutant or phosphorylated WT GC-A (Table 1).

No data containing the expected mass-to-charge ratio of the predicted phosphopeptide containing Ser-473 were identified in tryptic digests of GC-A (Schröter et al., 2010; Yoder et al., 2010). However, in single substrate concentration assays, Ala but not Glu substitutions for Ser-473 resulted in an approximately 20\% reduction in ANP-dependent activity when normalized to detergent-dependent activity (Yoder et al., 2012). In contrast, when GC activities of the individual 473-Ala and 473-Glu mutants were assessed by substrate-velocity assays, no difference in $V_{\max }$ or $K_{\mathrm{m}}$ values was observed between either mutant or phosphorylated WT-GC-A (Table 1).

Enzymatic Characterization of GC-A Mutants with Multiple Glutamate Substitutions. GC-A-6E, an enzyme containing Glu substitutions for Ser-497, Thr-500, Ser-502, Ser-506, Ser-510, and Thr-513, was stimulated by ANP about 10 -fold but was only about $20 \%$ as active as the phosphorylated WT enzyme (Potter and Hunter, 1999). Substituting an additional Glu for Ser-487 slightly increased $V_{\max }$ and decreased the $K_{\mathrm{m}}$. Adding the Ser-473-Glu substitution to GC-A-6E increased $V_{\max } 63 \%$ and decreased the $K_{\mathrm{m}} 4$-fold, but the $K_{\mathrm{m}}$ of GC-A-6E-S473E (GCA-7E) was still 2-fold higher than the phosphorylated WT enzyme (Fig. 2, A and B). Converting all eight sites to Glu to generate GC-A-6E-S473E-S487E (now named GC-A-8E) resulted in an enzyme that mimicked the enzymatic activity of the phosphorylated WT enzyme (Fig. 2C).

Phosphorylation Site Mutants Have WT-Like Concentration Responses. The effect of multiple phosphorylation site substitutions on ANP concentration responses was also determined. GC assays performed on membrane preparations from 293 cells transiently transfected with WT-GC-A, GC-A-6E, or GC-A-8E determined that the $\mathrm{EC}_{50}$ was not significantly different between either of the mutants and WT-GC-A (Fig. 3, A and B). The $\mathrm{EC}_{50}$ for ANP was also determined for WT-GC-A, GC-A-6E, GC-A-6E-S473E, GC-A6E-S487E, and GC-A-8E in live, transiently transfected 293 cells (Fig. 3, C and D). No differences in $\mathrm{EC}_{50}$ were observed between any of the GC-A enzymes, although cells expressing GC-A-6E and GC-A-6E-S487E had lower maximal cGMP levels and cells expressing GC-A-6E-S473E and GC-A-8E had higher maximal cGMP levels than cells expressing WT-GC-A.

A Negative Charge of Eight in the $N$ Terminus of the KHD Provides WT-Like GC-A Responsiveness. The effect of sequentially removing negative charge by substituting Ala for individual Glu in GC-A-8E was investigated. The substitutions were started at the C-terminal-most phosphorylation site. For instance, in Fig. 4A, 7E equals GC-A-8E with an E513A substitution, whereas 6E equals GC-A-8E with E513A and E510A substitutions. Substrate-velocity assays indicated that the first four substitutions resulted in a stepwise increase in the $K_{\mathrm{m}}$ without significantly affecting $V_{\max }$ (Fig. 4A). However, one additional Ala substitution to make GC-A-3E increased the $K_{\mathrm{m}} 4.6$-fold and the next Ala substitution reduced the $V_{\max } 6$-fold.

The effect of increasing negative charge to greater than -8 was also investigated by substituting Glu for Ser-494 or Ser494 and Ser-514, which are not naturally phosphorylated, in GC-A-8E to make GC-A-9E and GC-A-10E, respectively. However, the additional negative charge did not significantly affect the $K_{\mathrm{m}}$ or $V_{\max }$ values of GC-A (Fig. 4B). 


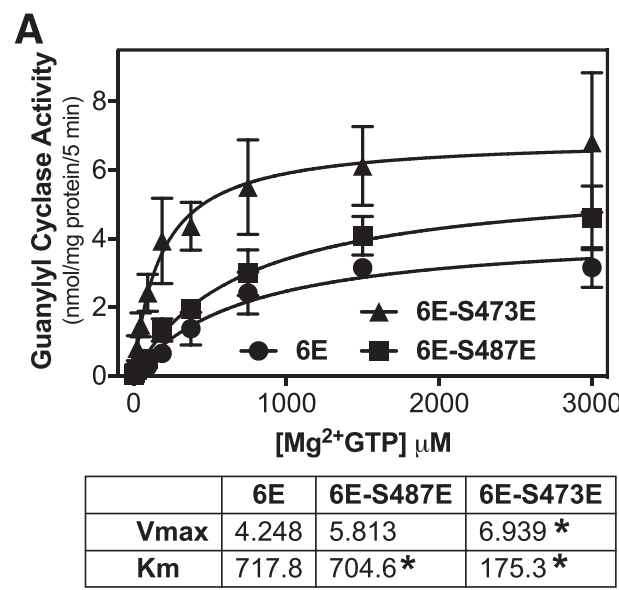

B
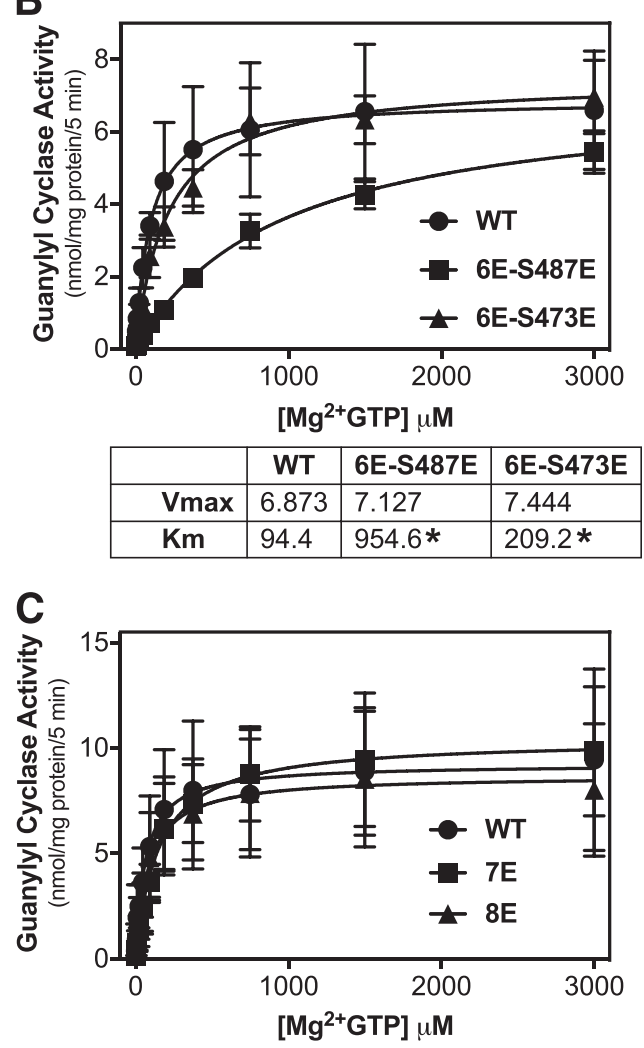

\begin{tabular}{|l|l|c|c|}
\hline & WT & 7E & 8E \\
\hline Vmax & 9.235 & 10.42 & 8.693 \\
\hline Km & 64.2 & $149.7 *$ & 86.07 \\
\hline
\end{tabular}

Fig. 2. Glutamate substitutions at Ser-473 and Ser-487 in GC-A-6E produce enzymes with similar characteristics to phosphorylated WT-GCA. 293 cells were transiently transfected with the indicated form of GC-A and assayed for GC activity for 5 minutes in the presence of $1 \mu \mathrm{M}$ ANP, $1 \mathrm{mM}$ ATP, and increasing concentrations of GTP. (A) Comparison between GC-A6E, GC-A-6E-S473E, and GC-A-6E-487E ( $n=2$ from two experiments). (B) Comparison between WT-GC-A, GC-A-6E-487E, and GC-A-6E-473E $(n=$ 3 from three experiments). (C) Comparison between WT-GC-A, GC-A-7E (GC-A-6E-473E), and GC-A-8E (GC-A-7E-487E) $(n=4$ from four experiments). $* P<0.0125$ (significantly different from WT-GC-A in B and $\mathrm{C}$ or GC-A-6E in A). Vertical bars in the symbols represent S.E.M.

Double Alanine Substitutions Dramatically Increased the $\boldsymbol{K}_{\mathrm{m}}$ and Modestly Decreased $\boldsymbol{V}_{\mathbf{m a x}}$. Next, we compared the effect of double Ala mutations for known phosphorylation sites to the effects of single Ala substitutions for GC-A
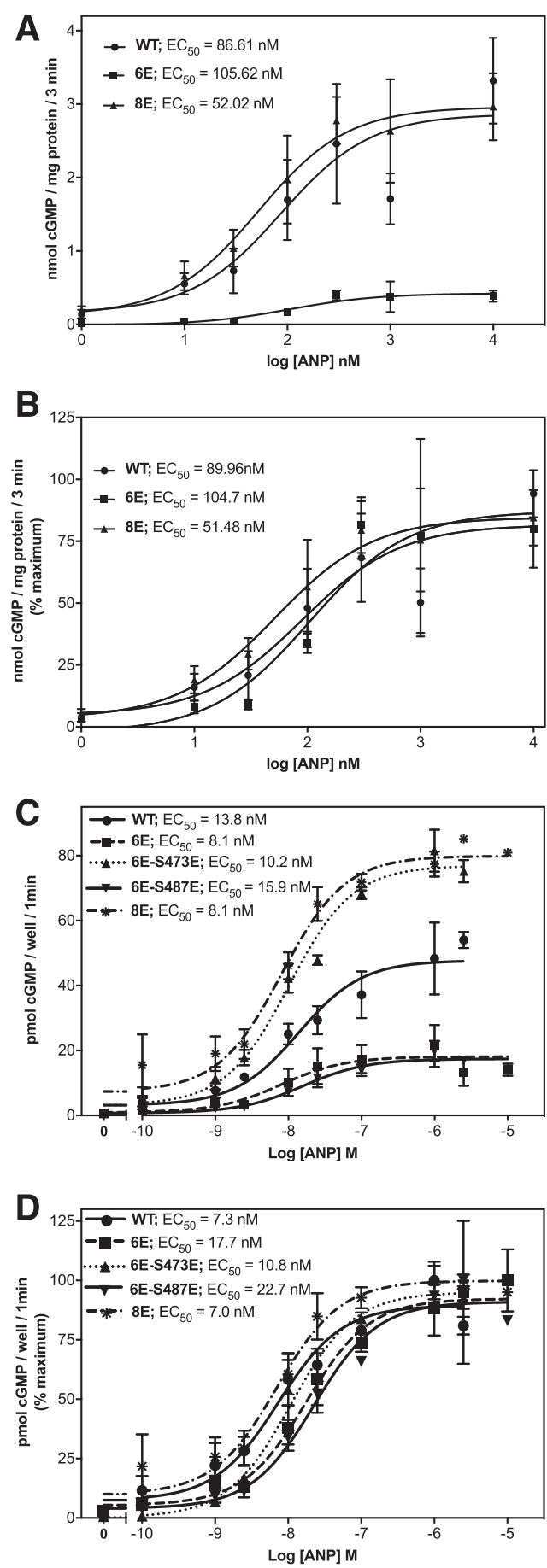

Fig. 3. GC-A-8E has a similar ANP dose response to phosphorylated WT-GCA. (A) 293 cells were transiently transfected with the indicated form of GC-A and assayed for GC activity for 3 minutes in the presence of $0.1 \mathrm{mM}$ GTP, $1 \mathrm{mM}$ ATP, and increasing concentrations of ANP $(n=6$ from three experiments). (B) Percentage of maximum activity for each construct ( $n=6$ from three experiments). (C) 293 cells were transiently transfected with the indicated form of GC-A. 119,000 cells/well were plated and assayed for ANP-dependent cGMP elevations in the medium after 1 minute in the presence of increasing concentrations of ANP $(n=2$ from two experiments). (D) The percentage of maximum activity is shown for each construct ( $n=2$ from two experiments). Vertical bars in the symbols represent the range of duplicate determinations.

phosphorylation sites shown in Table 1. Three double Ala or Glu mutants containing Ser- 497 and either Thr-500, Ser-510, or Thr-513 substitutions were analyzed (Fig. 5). Ala substitutions 


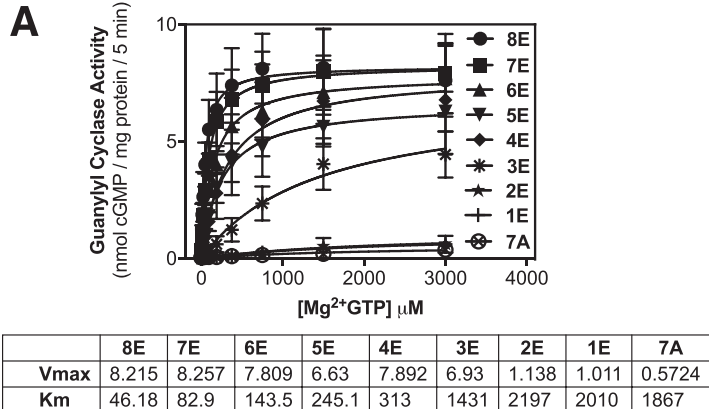

B

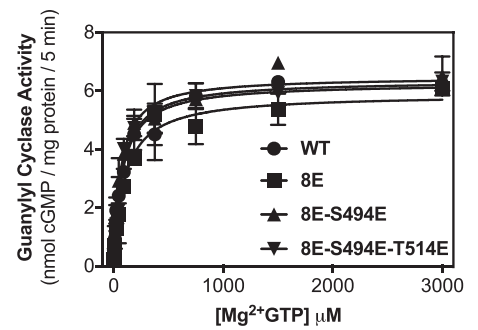

\begin{tabular}{|l|c|l|l|l|}
\hline & WT & 8E & 8E-S494E & 8E-S494E-T514E \\
\hline Vmax & 6.266 & 5.885 & 6.479 & 6.352 \\
\hline Km & 76.77 & 99.9 & 63.54 & 74.42 \\
\hline
\end{tabular}

Fig. 4. A threshold negative charge of eight is required for maximal GC-A activity. 293 cells were transiently transfected with the indicated form of GC-A and membranes from these cells were assayed for GC activity for 5 minutes in the presence of $1 \mu \mathrm{M}$ ANP, $1 \mathrm{mM}$ ATP, and increasing concentrations of GTP. (A) Comparison between GC-A-8E, GC-A-7E (GC-A-8E with 513A), GC-A-6E (GC-A-7E with 510A), GC-A-5E (GC-A-6E with 506A), GC-A-4E (GC-A-5E with 502A), GC-A-3E (GC-A-4E with 500A), GC-A-2E (GC-A-3E with 497A), GC-A-1E (GC-A-2E with 487A), and GC-A7A (WT-GC-A with 513A, 510A, 506A, 502A, 500A, 497A, 487S, and 473A) ( $n=2$ from two experiments). (B) Comparison between GC-A-8E, GC-A-8E$494 \mathrm{E}$, and GC-A-8E-494E and 514E ( $n=2$ from two experiments). Vertical bars in the symbols represent the range of duplicate determinations.

at Ser-497 and Thr-500 reduced the $V_{\max } 75 \%$ but increased the $K_{\mathrm{m}}$ 23-fold compared with the WT enzyme. From Table 1, Ala substitutions for Ser-497 and Thr-500 reduced $V_{\max }$ to $59 \%$ and $45 \%$ of the $V_{\max }$ for WT-GC-A. However, $V_{\max }$ and $K_{\mathrm{m}}$ values of the double Ala mutant were $25 \%$ and 23.3-fold more, respectively, of WT-GC-A. Adding the effect on $V_{\max }$ brought by single Ala substitutions at Ser-497 and Thr-500 results in a $96 \%$ reduction in $V_{\max }$ compared with WT (Table 1). Thus, the double Ala mutant has a close additive effect on $V_{\max }$. However, adding the effect on $K_{\mathrm{m}}$ brought by single Ala substitutions at Ser- 497 and Thr-500 results in a 5-fold increase in $K_{\mathrm{m}}$ (Table 1 ). Thus, the double Ala substitution at Ser- 497 and Thr-500 has a synergistic effect on the $K_{\mathrm{m}}$ of GC-A. In contrast, the corresponding double Glu mutant had no effect on $V_{\max }$ and only increased the $K_{\mathrm{m}}$ about 2-fold. Ala substitutions at Ser-497 and Ser-510 reduced $V_{\max } 86 \%$ and increased the $K_{\mathrm{m}} 46$-fold but, in this case, the corresponding Glu mutations did not completely restore activity to WT levels, although it was much more active than the corresponding Ala mutant. Ala substitutions for Ser-497 and Thr-513 did not significantly reduce the $V_{\max }$ but increased the $K_{\mathrm{m}}$ 70-fold. However, the corresponding double Glu mutant produced $V_{\max }$ and $K_{\mathrm{m}}$ values that were not different from the WT enzyme. Since the double Ala mutations had dramatically reduced $V_{\max }$ values, we loaded an equal amount of total protein and performed Western blots but found no differences in GC-A protein levels between the mutants and the WT enzyme (Fig. 5). Coomassie staining was performed on the same membrane after Western blotting to demonstrate equal protein loading (data not
A
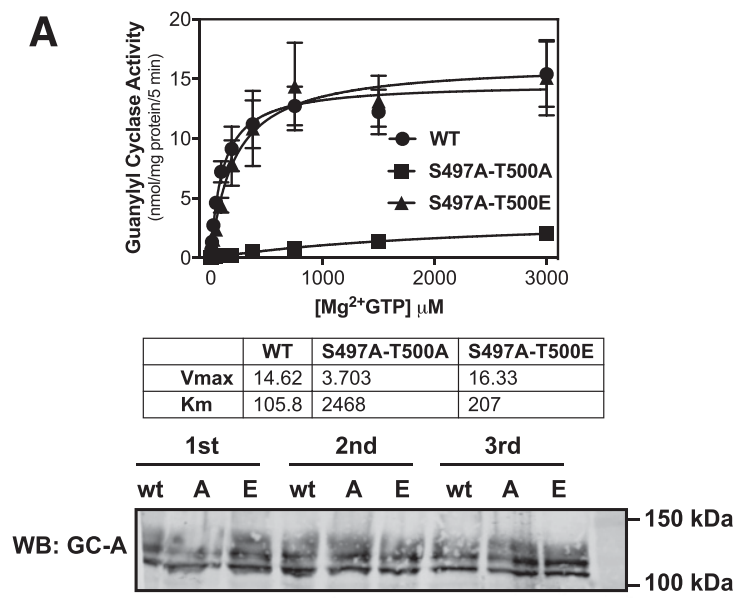

B
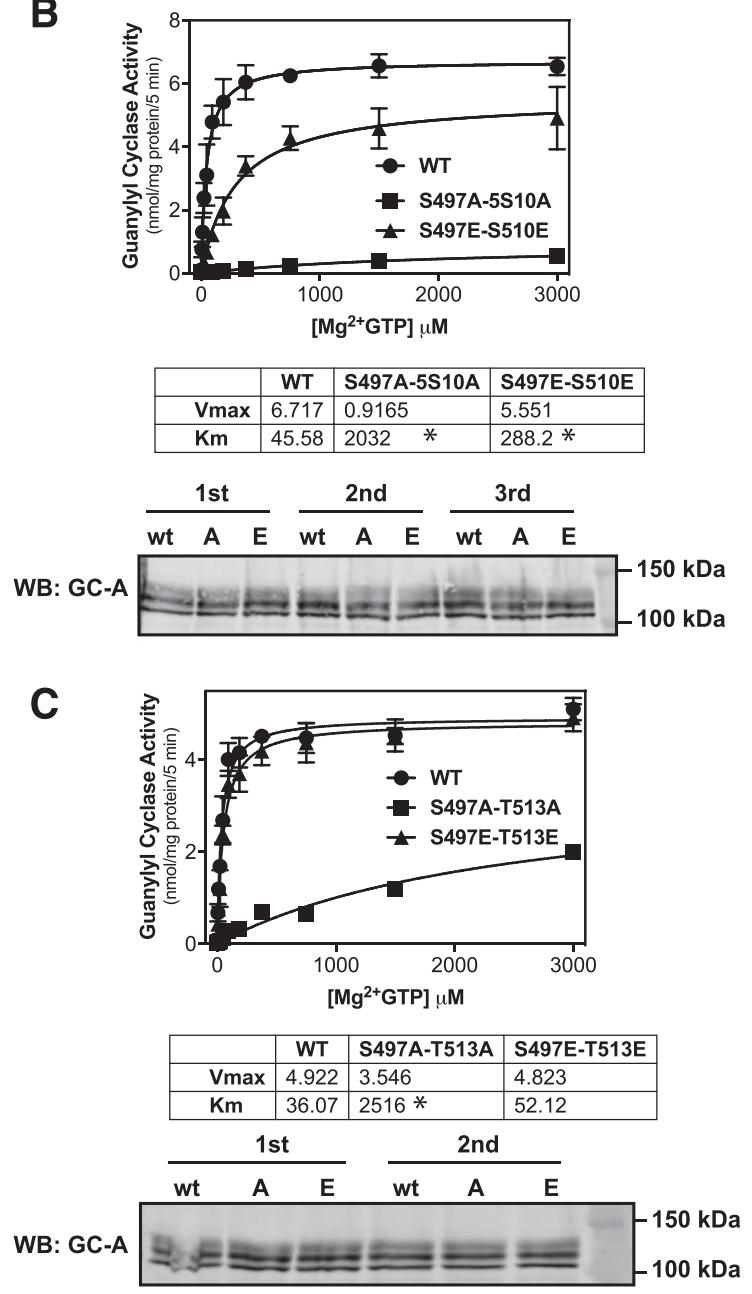

Fig. 5. Ala substitutions at Ser-497 and either Thr-500, Ser-510, or Thr513 have synergistic effects on the $K_{\mathrm{m}}$ for GC-A. 293 cells were transiently transfected with the indicated form of GC-A and membranes from these cells were assayed for GC activity for 5 minutes in the presence of $1 \mu \mathrm{M}$ ANP, $1 \mathrm{mM}$ ATP, and increasing concentrations of GTP. (A) Comparison between WT-GC-A, GC-A-S497A-T500A, and GC-A-S497E-T500E $(n=$ 3 from three experiments). (B) Comparison between WT-GC-A, GC-AS497A-S510A, and GC-A-S497E-S510E ( $n=3$ from three experiments). (C) Comparison between WT-GC-A, GC-A-S497A-T513A, and GC-A-S497ET513E ( $n=2$ from two experiments). $* P<0.0125$ (significantly different from WT-GC-A). Western blots indicate the amount of protein for each form of GC-A for each experiment. Vertical bars in the symbols represent the S.E.M. 
shown). In summary, all double Ala mutations had a synergistic effect on the $K_{\mathrm{m}}$ compared the increase in the $K_{\mathrm{m}}$ observed with
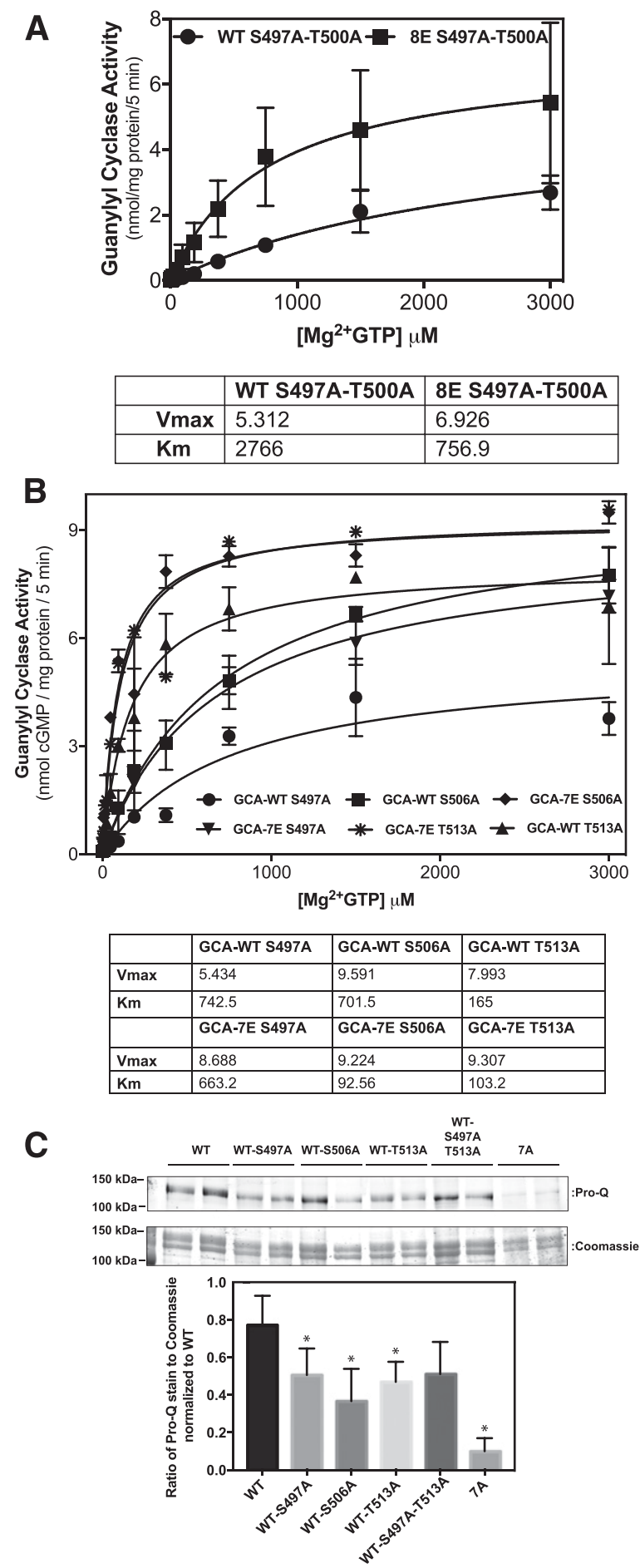

Fig. 6. Differential effects of phosphorylation site mutations on phosphorylated WT-GC-A and GC-A-8E. 293 cells were transiently transfected with the indicated form of GC-A and assayed for GC activity for 5 minutes in the presence of $1 \mu \mathrm{M}$ ANP, $1 \mathrm{mM}$ ATP, and increasing concentrations of GTP. (A) Comparison between GC-A-8E-S497A and T500A as well as GC-A-WT-S497A and T500A ( $n=2$ from two experiments). Vertical bars in the symbols represent the range of duplicate determinations. (B) Comparison between WT-GC-A-S497A, WT-GC-A-S506A, WT-GC-A-T513A, GC-A-7E-S497A, GC-A-7E-S506A, and GC-A-7E-T513A ( $n=2$ from two experiments). Vertical bars in the symbols represent the range of duplicate determinations. (C) Pro-Q Diamond and Coomassie staining comparing the single Ala substitutions shown in Table 1. In contrast, the effects on $V_{\max }$ were closer to additive.

Differential Effects of Phosphorylation Sites. The double Ala mutants had a synergistic negative effect on the $K_{\mathrm{m}}$ of the enzyme and an additive or slightly greater than additive effect on the $V_{\max }$. One explanation for these drastic effects is that double Ala mutations promote the dephosphorylation of other sites in addition to the mutated sites. To test this possibility, Ala substitutions at both Ser-497 and Thr-500 were introduced into either GC-A-8E or into the phosphorylated WT enzyme. Consistent with the Ala substitutions affecting more than the modified site, the Ala-substituted version of WT-GC-A had much less activity than the Alasubstituted version of GC-A-8E (Fig. 6A). The effect of single Ala mutations was also compared between the phosphorylated WT form of GC-A or GC-A-8E and, again, the GC-A-8E mutants had higher enzymatic activity than the corresponding mutations introduced into WT-GC-A, which is consistent with the idea that one site affects the phosphorylation of other sites (Fig. 6B). In agreement with the synergistic effect on protein phosphorylation, Pro-Q phosphate and Coomassie protein analysis indicated that Ala substitutions for Ser-497, Ser-506, Thr-513, and the double mutant Ser-497 and Thr-513 reduced the phosphate content of GC-A more than would be expected if the mutations only eliminated only one phosphate. For instance, GC-A has seven chemically identified phosphorylation sites but the single S506A mutation decreased the phosphate content of GC-A by about half, which suggests that the loss of phosphorylation of Ser-506 is affecting the phosphorylation of other sites as well.

\section{Discussion}

Phosphorylation affects proteins by facilitating dockings interactions that involve steric and charge interactions between different proteins or by causing unimolecular structural changes through salt bridge formation that result solely from charge interactions (Hunter, 2012). Here, we report that replacement of phosphorylated Ser and Thr in GC-A with Glu, a sterically smaller moiety with a negative charge, completely mimics the functional characteristics of the phosphorylated WT enzyme. Hence, we conclude that phosphorylation is required for hormonal activation of GC-A solely due to charge interactions. Furthermore, we suggest that the critical amount of negative charge in and around subdomain I of the KHD is approximately -8 and that reductions in negative charge in this area reduce activity primarily by raising the $K_{\mathrm{m}}$.

Reductions in enzyme activity were observed for all Ala substitutions of phosphorylation sites with the exception of Ser-473 and Ser-487. Initial single substrate assays revealed relatively small reductions in activity for the 487-Glu and no effect for 487-Ala. It is unclear why no significant differences were observed in the substrate-velocity assays. However, previous assays evaluated activity differences when expressed

phosphate and protein values for WT-GC-A, WT-GC-A-S497A, WT-GC-AS506A, WT-GC-A-T513A, WT-GC-A-S497A and T513A, and WT-GC-A7A. The ratio of the intensity of Pro-Q Diamond staining to Coomassie staining was normalized to WT ( $n=4$ from four independent immunoprecipitations). $* P<0.05$ (significantly different from WT-GC-A). Vertical bars in the symbols represent the S.E.M. 
as percent of detergent-dependent GC activity (Yoder et al., 2010), and detergent normalization of activity was not employed in the current study. No change in the substrate concentration curves was observed for Ala or Glu mutants of Ser-473 either. This differs from the Ala substitution at the corresponding residue in GC-B, Ser-489, which increased the $K_{\mathrm{m}}$ of the GC-B mutant 5 -fold (Yoder et al., 2012). The reason for the differing effects of the single Ala substitution of this juxtamembrane site in GC-A and GC-B is not known but the Ser-489-Ala mutant was compared with the Ser-489-Glu mutant, which has a slightly lower $K_{\mathrm{m}}$ than the WT enzyme and likely exaggerated the difference. It is also possible that phosphorylation of Ser-487 in GC-A may be a factor, since this site is not conserved in GC-B.

For the first time, we present evidence for phosphorylation site interdependence in GC-A. Single or double Ala mutations for known phosphorylation sites had a much greater effect in the WT phosphorylated enzyme than in the glutamate- substituted form of GC-A (Fig. 6), which is consistent with one phosphorylation site affecting other phosphorylation sites. For example, Ala substitution at Ser-506 had a much greater effect on WT-GCA than GC-A-8E, suggesting that negative charge at Ser-506 is important for the phosphorylation of other sites. In agreement with this observation, Pro-Q analysis revealed that the S506A mutation decreased receptor phosphate content by about $50 \%$, which is more than could be explained by the loss of a single site. However, the location of the charge is also important, since the mutation of Ser-497 to Ala markedly reduced the activity of both WT-GC-A and GC-A-8E

Based on the data reported here and elsewhere, we propose a model in which the phosphorylation of GC-A causes a conformational change in the KHD that is required for transmission of the hormone binding signal. We predict that when GC-A is in its dephosphorylated state, its KHD is unordered due to the lack of negatively charged residues that normally

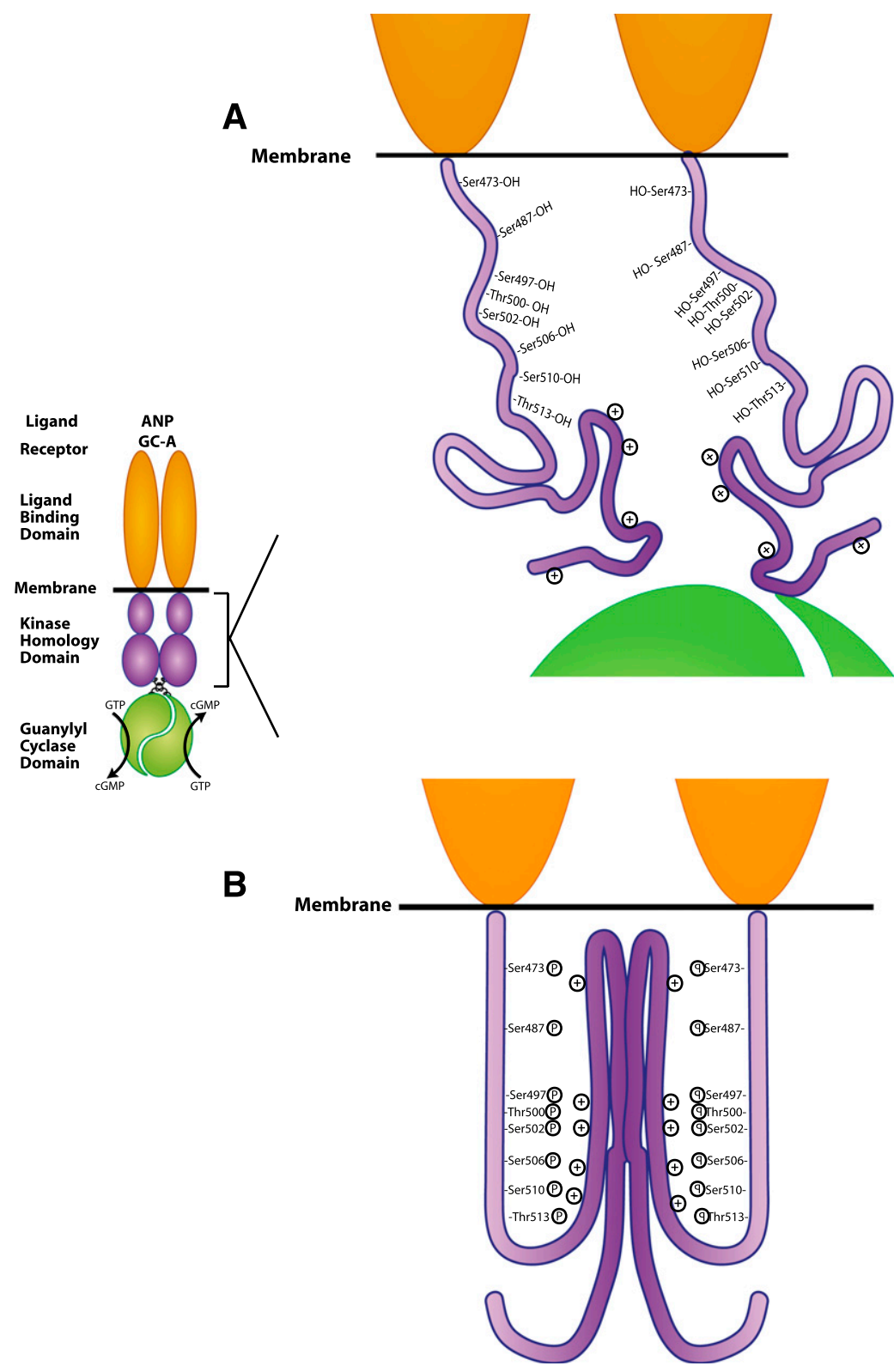

Fig. 7. Model of phosphorylation-dependent ANP-stimulation of GC-A. (A) When GC-A is in its dephosphorylated state, the KHD is unordered and does not allow transmission of the ANP binding to the catalytic domain. (B) The increased negative charge resulting from the phosphorylation sites provide a concentrated region of negative charge that interacts with positive residues within the KHD, which provides a ridged and ordered structure that allows the rotational force elicited by ANP binding to be transferred to the catalytic domain to decrease the $K_{\mathrm{m}}$ and increase $V_{\max }$. 
form salt bridges with positively charged Arg and Lys residues (Fig. 7A). When these salt bridges are formed (Fig. 7B), a ridged and ordered structure is formed. Crystal structure studies comparing unbound receptor to ANP bound receptor reveals that ANP binding causes the two receptor monomers to undergo an intermolecular twist of about 30 degrees (Ogawa et al., 2004). We hypothesize that phosphorylation provides a rigid KHD downstream of the extracellular domain that allows the rotational force caused by ANP binding to be transmitted to the catalytic domain to increase substrate affinity.

In conclusion, we describe for the first time, to our knowledge, a dephosphorylated form of GC-A called GC-A-8E that has the same activity as the phosphorylated WT enzyme. Although much has been reported regarding the effects of phosphorylation of GC-A in cell culture (Potter et al., 2006), nothing is currently known about the role of phosphorylation in the control of GC-A in a physiologic setting. Recent "knockin" studies revealed a critical role of GC-B dephosphorylation in the resumption of meiosis in the oocyte (Shuhaibar et al., 2016). To identify physiologic functions that are regulated by changes in GC-A phosphorylation, we collaborated with Dr. Laurinda Jaffe's group to generate homozygous GC-A ${ }^{8 \mathrm{E} / 8 \mathrm{E}}$ mice. Potential phenotypic differences in the GC-A ${ }^{8 \mathrm{E} / 8 \mathrm{E}}$ compared with GC-A ${ }^{\mathrm{WT} / \mathrm{WT}}$ mice are reduced blood pressure (Lopez et al., 1995), reduced cardiac hypertrophy (Knowles et al., 2001), and/or increased fat metabolism (Bordicchia et al., 2012). Finally, whether changes in GC-A phosphorylation affect disease processes is not known. However, we recently found that the Ser-488-Pro mutation in GC-B (Miura et al., 2014) only activates the phosphorylated form of the enzyme (D. M. Dickey et al., manuscript in preparation), which suggests that similar scenarios may exist for GC-A as well.

\section{Authorship Contributions}

Participated in research design: Otto, Potter.

Conducted experiments: Otto, McDowell, Dickey.

Performed data analysis: Otto, Dickey, Potter.

Wrote or contributed to the writing of the manuscript: Otto, Potter.

\section{References}

Abbey SE and Potter LR (2002) Vasopressin-dependent inhibition of the C-type natriuretic peptide receptor, NPR-B/GC-B, requires elevated intracellular calcium concentrations. J Biol Chem 277:42423-42430.

Abbey SE and Potter LR (2003) Lysophosphatidic acid inhibits C-type natriuretic peptide activation of guanylyl cyclase-B. Endocrinology 144:240-246.

Abbey-Hosch SE, Cody AN, and Potter LR (2004) Sphingosine-1-phosphate inhibits C-type natriuretic peptide activation of guanylyl cyclase B (GC-B/NPR-B). Hypertension 43:1103-1109.

Antos LK, Abbey-Hosch SE, Flora DR, and Potter LR (2005) ATP-independent activation of natriuretic peptide receptors. J Biol Chem 280:26928-26932.

Bordicchia M, Liu D, Amri EZ, Ailhaud G, Dessì-Fulgheri P, Zhang C, Takahashi N, Sarzani R, and Collins S (2012) Cardiac natriuretic peptides act via p38 MAPK to induce the brown fat thermogenic program in mouse and human adipocytes. J Clin Invest 122:1022-1036.

Bryan PM, Smirnov D, Smolenski A, Feil S, Feil R, Hofmann F, Lohmann S, and Potter LR (2006) A sensitive method for determining the phosphorylation status of natriuretic peptide receptors: cGK-Ialpha does not regulate NPR-A. Biochemistry 45:1295-1303.

Bryan PM, Xu X, Dickey DM, Chen Y, and Potter LR (2007) Renal hyporesponsiveness to atrial natriuretic peptide in congestive heart failure results from reduced atrial natriuretic peptide receptor concentrations. Am J Physiol Renal Physiol 292 F1636-F1644.

Dickey DM, Burnett JC, Jr, and Potter LR (2008) Novel bifunctional natriuretic peptides as potential therapeutics. J Biol Chem 283:35003-35009.

Dickey DM, Dries DL, Margulies KB, and Potter LR (2012) Guanylyl cyclase (GC)-A and GC-B activities in ventricles and cardiomyocytes from failed and non-failed human hearts: GC-A is inactive in the failed cardiomyocyte. $\mathrm{J} \mathrm{Mol} \mathrm{Cell} \mathrm{Cardiol} \mathbf{5 2}$ 727-732.

Dickey DM, Flora DR, Bryan PM, Xu X, Chen Y, and Potter LR (2007) Differential regulation of membrane guanylyl cyclases in congestive heart failure: natriuretic peptide receptor (NPR)-B, not NPR-A, is the predominant natriuretic peptide receptor in the failing heart. Endocrinology 148:3518-3522.

Egbert JR, Shuhaibar LC, Edmund AB, Van Helden DA, Robinson JW, Uliasz TF, Baena V, Geerts A, Wunder F, Potter LR, et al. (2014) Dephosphorylation and inactivation of NPR2 guanylyl cyclase in granulosa cells contributes to the LH-induced decrease in cGMP that causes resumption of meiosis in rat oocytes. Development 141:3594-3604.

Hubers SA and Brown NJ (2016) Combined angiotensin receptor antagonism and neprilysin inhibition. Circulation 133:1115-1124.

Hunter T (2012) Why nature chose phosphate to modify proteins. Philos Trans $R$ Soc Lond B Biol Sci 367:2513-2516.

Joubert S, Labrecque J, and De Léan A (2001) Reduced activity of the NPR-A kinase triggers dephosphorylation and homologous desensitization of the receptor. Biochemistry 40:11096-11105.

Knowles JW, Esposito G, Mao L, Hagaman JR, Fox JE, Smithies O, Rockman HA and Maeda N (2001) Pressure-independent enhancement of cardiac hypertrophy in natriuretic peptide receptor A-deficient mice. J Clin Invest 107:975-984.

Koller KJ, Lipari MT, and Goeddel DV (1993) Proper glycosylation and phosphorylation of the type A natriuretic peptide receptor are required for hormonestimulated guanylyl cyclase activity. J Biol Chem 268:5997-6003.

Kuhn M (2016) Molecular physiology of membrane guanylyl cyclase receptors. Physiol Rev 96:751-804.

Lopez MJ, Wong SK, Kishimoto I, Dubois S, Mach V, Friesen J, Garbers DL, and Beuve A (1995) Salt-resistant hypertension in mice lacking the guanylyl cyclase-A receptor for atrial natriuretic peptide. Nature 378:65-68.

Miura K, Kim OH, Lee HR, Namba N, Michigami T, Yoo WJ, Choi IH, Ozono K, and Cho TJ (2014) Overgrowth syndrome associated with a gain-of-function mutation of the natriuretic peptide receptor 2 (NPR2) gene. Am J Med Genet A 164A: 156-163.

Müller D, Cortes-Dericks L, Budnik LT, Brunswig-Spickenheier B, Pancratius M, Speth RC, Mukhopadhyay AK, and Middendorff R (2006) Homologous and lysophosphatidic acid-induced desensitization of the atrial natriuretic peptide receptor, guanylyl cyclase-A, in MA-10 Leydig cells. Endocrinology 147:2974-2985.

Ogawa H, Qiu Y, Ogata CM, and Misono KS (2004) Crystal structure of hormonebound atrial natriuretic peptide receptor extracellular domain: rotation mechanism for transmembrane signal transduction. J Biol Chem 279:28625-28631.

Potter LR (1998) Phosphorylation-dependent regulation of the guanylyl cyclaselinked natriuretic peptide receptor B: dephosphorylation is a mechanism of desensitization. Biochemistry 37:2422-2429.

Potter LR (2011) Guanylyl cyclase structure, function and regulation. Cell Signal 23: 1921-1926

Potter LR, Abbey-Hosch S, and Dickey DM (2006) Natriuretic peptides, their receptors, and cyclic guanosine monophosphate-dependent signaling functions. Endocr Rev 27:47-72.

Potter LR and Garbers DL (1992) Dephosphorylation of the guanylyl cyclase-A receptor causes desensitization. J Biol Chem 267:14531-14534

Potter LR and Garbers DL (1994) Protein kinase C-dependent desensitization of the atrial natriuretic peptide receptor is mediated by dephosphorylation. J Biol Chem 269:14636-14642.

Potter LR and Hunter T (1998a) Identification and characterization of the major phosphorylation sites of the B-type natriuretic peptide receptor. J Biol Chem $\mathbf{2 7 3}$ : 15533-15539.

Potter LR and Hunter T (1998b) Phosphorylation of the kinase homology domain is essential for activation of the A-type natriuretic peptide receptor. Mol Cell Biol 18 2164-2172.

Potter LR and Hunter T (1999) A constitutively "phosphorylated" guanylyl cyclaselinked atrial natriuretic peptide receptor mutant is resistant to desensitization. Mol Biol Cell 10:1811-1820.

Potter LR and Hunter T (2000) Activation of protein kinase C stimulates the dephosphorylation of natriuretic peptide receptor-B at a single serine residue: a possible mechanism of heterologous desensitization. J Biol Chem 275:3109931106.

Potter LR and Hunter T (2001) Guanylyl cyclase-linked natriuretic peptide receptors: structure and regulation. $J$ Biol Chem 276:6057-6060.

Robinson JW and Potter LR (2012) Guanylyl cyclases A and B are asymmetric dimers that are allosterically activated by ATP binding to the catalytic domain. Sci Signal 5:ra65.

Schröter J, Zahedi RP, Hartmann M, Gassner B, Gazinski A, Waschke J, Sickmann A, and Kuhn M (2010) Homologous desensitization of guanylyl cyclase A, the receptor for atrial natriuretic peptide, is associated with a complex phosphorylation pattern. FEBS J 277:2440-2453.

Shuhaibar LC, Egbert JR, Edmund AB, Uliasz TF, Dickey DM, Yee SP, Potter LR, and Jaffe LA (2016) Dephosphorylation of juxtamembrane serines and threonines of the NPR2 guanylyl cyclase is required for rapid resumption of oocyte meiosis in response to luteinizing hormone. Dev Biol 409:194-201.

Yoder AR, Robinson JW, Dickey DM, Andersland J, Rose BA, Stone MD, Griffin TJ, and Potter LR (2012) A functional screen provides evidence for a conserved, regulatory, juxtamembrane phosphorylation site in guanylyl cyclase a and B. PLoS One 7:e36747.

Yoder AR, Stone MD, Griffin TJ, and Potter LR (2010) Mass spectrometric identification of phosphorylation sites in guanylyl cyclase A and B. Biochemistry 49: 10137-10145.

Address correspondence to: Lincoln R. Potter, Department of Biochemistry, Molecular Biology, and Biophysics, University of Minnesota Medical School, 6-155 Jackson Hall, 321 Church Street SE, Minneapolis, MN 55455. E-mail: potter@umn.edu 